\title{
Atividade física contra redução da massa óssea induzida por glicocorticoides
}

\section{Physical activity against reduction of mass bone induced for glucocorticoids}

\author{
Rodolfo Pessato Timóteo'; Dirce Sofia Fabbri de Almeida Verde dos Santos ${ }^{2}$ \\ ${ }^{1}$ Fisioterapeuta - Uniube - Uberaba/MG \\ 2 Farmacêutica - Uniube - Uberaba/MG \\ Endereço para correspondência \\ Rodolfo Pessato Timóteo \\ R. Manoel Gonçalves de Freitas, n 105, Bairro Cássio Resende I \\ 38080-330 - Uberaba - MG [Brasil] \\ rodolfo_pessa@hotmail.com
}

\begin{abstract}
Resumo
A massa óssea é alterada por vários fatores, chegando à osteoporose em decorrência da ação dos glicocorticoides, medicamentos usados em várias doenças como as autoimunes. A prática de atividades físicas já é coadjuvante no tratamento e prevenção da osteoporose pós-menopáusica e senil, e pode trazer benefícios diretos e indiretos para diminuir os efeitos colaterais dos glicocorticoides. O objetivo deste trabalho foi realizar levantamento e análise da bibliografia para encontrar, na literatura, argumentos que confirmem a utilização dos exercícios físicos como prevenção e tratamento dos efeitos maléficos dos glicocorticoiwdes em relação à massa óssea. Há concordância quanto ao benefício das atividades físicas em pacientes usuários crônicos de glicocorticoides, tanto no que se refere à massa óssea quanto à minimização dos efeitos da síndrome metabólica. Concluiu-se que os resultados dos exercícios físicos sobre o cortisol endógeno e exógeno não são totalmente explicados, pois necessitam de mais pesquisas sobre o assunto e a respeito da correlação dos benefícios fisioterapêuticos em pacientes usuários de glicocorticoides.
\end{abstract}

Descritores: Atividade física; Glicocorticoides; Massa óssea.

\begin{abstract}
The bone mass is altered because of many factors, having osteoporosis resulted from the action of glucocorticoids, medicines used in many diseases, like in the self immune ones; physical exercise is already a possibility in the treatment and prevention of postmenopausal and senile osteoporosis, and can benefit directly and indirectly the collateral effects of the glucocorticoids. The aim of this article was to find in the literature arguments that confirm the contribution of the exercises as a prevention and treatment to the harmful effects of the glucocorticoids in relation to the bone density. There is an agreement about the benefits of the physical activities in chronic user glucocorticoid patients not only in relation to the bone density but also to minimize the effects of the metabolic syndrome. The effects of the physical exercises about the endogenous and exogenous cortisol are not totally explained, demanding thus, more researches, such as the correlation of the physiotherapy benefits in chronic user glucocorticoids patients.
\end{abstract}

Key words: Bone mass; Glucocorticoids; Physical activity. 


\section{Introdução}

A fisiologia do sistema esquelético é alterada como efeito colateral comum decorrente da ação dos glicocorticoides (GC) que são discutidos quanto ao seu uso, por exercer efeitos sobre os sistemas orgânicos em razão de suas ações diversificadas, que resultam na diminuição da massa óssea pela redução de rigidez e força, chegando até mesmo à osteoporose. Atualmente, é a causa mais frequente de osteoporose secundária (1, $2,3,4,5,6,7)$. O efeito da diminuição da massa óssea foi descrito há mais de meio século por Harvey Cushing (1932). Por sua vez, as fraturas decorrentes desse processo são bastante comuns, principalmente no esqueleto axial. Apesar da escassez de relatos, essas fraturas ocorrem entre 30 e $50 \%$ dos pacientes que fazem uso de GC por mais de 6 meses. Todas as pessoas são susceptíveis à perda óssea induzida por esses medicamentos; no entanto, mulheres na pós-menopausa e crianças são particularmente mais vulneráveis ${ }^{8,9}$.

O tratamento da osteoporose induzida por GC pode ser feito com cálcio, vitamina $\mathrm{D}$, calcitonina, biofosfonatos e fluoreto de sódio. A abordagem terapêutica mais apropriada é o uso das mínimas doses necessárias e, dependendo da doença em questão, estão indicados GCs inalatórios, que têm menor repercussão sobre o metabolismo ósseo. Apesar de a terapêutica medicamentosa ser obviamente um tratamento contra os efeitos colaterais dos GCs, ainda não provou sua eficácia total ${ }^{8,10,11}$.

Pesquisas como as de Carvalho ${ }^{12}$ e Haach ${ }^{13}$ vêm demonstrando o efeito benéfico do ultra-som pulsado de baixa freqüência para benefício da massa óssea, assemelhando os resultados com alguns treinamentos físicos em ratas osteopênicas.

A indicação de exercícios regulares, orientados de forma correta, também faz parte do tratamento, já que estimula intrinsecamente a mineralização em decorrência do efeito piezoelétrico, além dos benefícios fisiológicos, que reagem, de forma positiva, para a estimulação e conservação dos osteoblastos por meio das adaptações geradas ${ }^{14,15,16}$.
O objetivo, neste trabalho, foi realizar levantamento e análise da bibliografia para encontrar na literatura argumentos que confirmem a contribuição do exercício físico para prevenção e tratamento dos efeitos maléficos dos GCs na massa óssea.

\section{Revisão da literatura}

\section{Ação dos glicocorticoides sobre a massa óssea}

Em um adulto normal, em estado emocional equilibrado (ausência de estresse), são produzidos de 10 a 20 mg dia de cortisol (também denominado composto F) em ritmo circadiano, que atinge valores áximos nas primeiras horas da manhã.

Esse hormônio liga-se à maior parte dos tipos de células do corpo humano, afetando a secreção de diversos hormônios. O cortisol endógeno atravessa a membrana lipoprotéica das células, ligando-se a receptores citosólicos e exercendo sua ação no interior do núcleo, interagindo com o DNA (ação genômica) ou com outras proteínas implicadas no processo de transcrição (ação não genômica, específica ou inespecífica). Um terceiro mecanismo envolvido, independentemente de genoma, foi observado pelos efeitos in vitro na respiração, na síntese de proteínas e na ação da $\mathrm{Na}^{+}$- $\mathrm{K}^{+}$- ATPase e Ca ${ }^{2+}$ - ATPase em timócitos ${ }^{17,18}$.

O principal cortisol endógeno, a hidrocortisona, afeta diretamente o metabolismo da glicose, das proteínas e dos ácidos graxos livres, por promover o fracionamento da proteína em aminoácido em todas as células do organismo, com exceção do fígado.

Os GCs sintéticos são obtidos do ácido fólico (retirado de bovinos) ou de algumas plantas das famílias das Liliaceae e Dioscoreaceae. Esses medicamentos administrados de forma oral agem rapidamente no organismo em razão da sua intensidade de ligação com proteínas ${ }^{18}$.

As ações dos esteroides sintéticos assemelham-se à do cortisol endógeno, porém variam apenas em intensidade, alterando, em muitas ve- 
zes, o suprimento energético do sistema nervoso central. A potência anti-inflamatória dos derivados dos GCs varia de acordo com as diferenças de ação, duração, efetividade intrínseca e sítio de ação, o que refletirá na intensidade dos efeitos colaterais ${ }^{10}$. Por sua vez a redução da massa óssea pelo uso de GC ocorre basicamente pela ação catabólica da matriz óssea, por diminuição da síntese e liberação protéica e formação de RNA ${ }^{10,18,19}$.

\section{Fisiopatologia}

A perda da massa óssea causada pelos GCs é dividida didaticamente em direta e indireta. Os efeitos diretos estão relacionados à ação dessa droga sobre as células ósseas e suas funções, e os indiretos, sobre o metabolismo do cálcio e vitamina D, a secreção dos hormônios sexuais e a produção de prostaglandinas, citocinas e fatores de crescimento ${ }^{8,10,20,21}$.

Os efeitos diretos sobre a formação óssea e a função osteoblástica são gerados pela diminuição da osteoblastogênese por volta de 48 horas da exposição ao medicamento, diminuindo a quantidade de células capazes de sintetizar colágeno ósseo, bem como pela promoção de apoptose dos osteoblastos e osteócitos, sendo esta última mais relevante.

Os GCs têm ações complexas sobre a expressão gênica das células esqueléticas, dependendo do estágio de diferenciação, crescimento e ação dos osteoblastos ${ }^{10}$. Esses agentes têm efeitos paradoxais, pois agem sobre as funções do RNA, impedindo que os pré-osteoblastos se transformem em osteoblastos maduros. Chega-se a esse resultado supostamente pela ação genética do próprio medicamento, o que impossibilita a formação da proteína osteocalcina, assim como (ou paralelamente) do colágeno tipo 1, com a possibilidade de os receptores citoplasmáticos se envolverem no processo. Além de diminuírem a síntese, os GCs aumentam a degradação do colágeno por meio do aumento da expressão das colagenases e da inibição da expressão do inibidor tecidual das metaloproteinases.
De acordo com Azevedo e Szejnfeld ${ }^{10}$, estudos de histologia em biópsia de pacientes tratados com GC mostraram que, em decorrência de sua ação, a superfície do osteoide, embora aumentada, estava com espessura diminuída.

Ainda entre os fatores diretos, os efeitos sobre a reabsorção óssea podem ser observados in vivo por meio do aumento da atividade osteoclástica, com aumento da quantidade de osso reabsorvido em cada sítio. Os GCs também podem aumentar a aderência dos macrófagos ao osso por modificarem a superfície dos oligossacárides, facilitando a ação osteoclástica ${ }^{8}$.

Já entre os fatores indiretos, a absorção intestinal e excreção renal do cálcio e fósforo são gerados pela diminuição do transporte intestinal transcelular de cálcio, independentemente da quantidade de vitamina D e contribuem para o aparecimento de hiperparatireoidismo secundário. A alteração do Hormônio da paratireoide (PTH) pode gerar fosfatase alcalina, síntese de colágeno, descarboxilação do citrato e aumento da sensibilidade tubular renal ao $\mathrm{PTH}^{8,10}$.

A secreção dos hormônios sexuais é afetada por efeitos que inibem a liberação hipofisária das gonadotrofinas, a secreção ovariana e testicular de estrógeno, a testosterona e a secreção adrenal de androstenediona e de hidroepiandrosterona. Em decorrência da ação anabólica desses esteroides sexuais, sua inibição causa agravo da osteoporose.

Os efeitos dos GCs nas prostaglandinas, nas citocinas e nos fatores de crescimento diminuem a produção de prostaglandina E2 no osso, cuja consequência principal é estimular a síntese de proteínas colágenas e não colágenas. Esse parece ser somente um mecanismo coadjuvante na osteoporose induzida por GC, uma vez que a perda óssea não pode ser reproduzida por drogas que inibem a síntese de prostaglandinas, como a indometacina.

Outro efeito indireto dos GCs sobre o osso envolve síntese, liberação e ligação ao receptor ou a proteínas de ligação dos fatores de crescimento produzidos pelas células ósseas. Os fatores de crescimento semelhantes à insulina (IGF) 
são importantes na manutenção da matriz óssea. Um aumento de 50 a $100 \%$ acima dos valores de normalidade do PTH é verificado em pacientes durante um período prolongado do uso de GC. Essa elevação foi afirmada com base na diminuição do cálcio plasmático e na ação direta sobre a paratireoide ${ }^{8}$.

\section{Exercício físico e massa óssea}

Os benefícios que as atividades físicas promovem nos sistemas do corpo humano são bem conhecidos; por sua vez, a prática de exercícios por usuários crônicos de GC serviria de meio de prevenção ou tratamento das consequências da síndrome metabólica gerada, resultando em hipertensão arterial, diabetes mellitus, aumento da gordura corporal em regiões específicas (rosto, região centrípeta abdominal e dorso) e diminuição drástica da massa muscular estriada esquelética.

$\mathrm{O}$ efeito benéfico da atividade física para a saúde óssea do paciente que faz uso crônico dos GCs decorre da responsividadde dinâmica do tecido ósseo à demanda funcional que lhe é imposta, o que gera alterações de sua massa e resistência. Essas mudanças resultam da força gravitacional e da ação intensa dos músculos ligados aos ossos. A resposta adaptativa do osso dependerá, portanto, da magnitude da carga e da frequência de aplicação, as quais, sendo regularmente repetidas, desencadeiam efeitos osteogênicos. Essa resposta adaptativa compreende a biomecânica natural do osso já postulada por Julius Wolff'22, 23, 24, 25, 26, reação confirmada por Silva et al. ${ }^{15}$ e Carvalho ${ }^{12}$ com base em pesquisas que provaram o aumento dos números de osteoblastos a partir de séries de estímulos de cargas locais. Cerca de $80 \%$ da massa óssea responde pela variação na força aplicada, mas outros fatores como geometria óssea, arquitetura interna e propriedades mecânicas também afetam a força do osso; portanto, o estresse contínuo provocado pelo exercício físico resulta em adaptações morfológicas, tais como aumento da espessura cortical e maior conteúdo ósseo na inserção musculotendí- nea. De acordo com Silva et al. ${ }^{15}$, os mecanismos pelos quais o esqueleto responde ao treinamento físico ainda não estão totalmente compreendidos.

Embora existam controvérsias na literatura a respeito das formas de adaptação do tecido ósseo exposto a cargas externas, o impacto do exercício físico sobre a massa óssea é relevante durante a adolescência, quando o pico dessa massa está por ser alcançado. Além de todos os efeitos diretos sobre a massa óssea, o exercício físico atua também de forma positiva, beneficiando o sistema cardiovascular e o endócrino $27,28,29,30,31$.

\section{Conclusão}

O efeito da diminuição da massa óssea foi confirmado nas literaturas pesquisadas que ditam sobre o assunto, concordando que o osso trabecular é atingido com mais intensidade por uma série de ações diretas e indiretas que inibem basicamente o equilíbrio da unidade multicelular básica (UMB).

Borba et al. ${ }^{8}$, Gennari ${ }^{21}$ e Azevedo et al. ${ }^{10}$, autores que ditam especificadamente sobre a diminuição da massa óssea e osteoporose causada por GC, correlacionam os efeitos diretos e indiretos e afirmam a importância da profilaxia principalmente para pacientes que farão uso crônico dos anti-inflamatórios esteroidais.

A maneira básica e natural de conseguir resultados quanto ao aumento da massa óssea, seria por meio do exercício físico (Lei de Wolff e reequilíbrio de UMB), o que geraria efeitos positivos para prevenção ou mesmo tratamento dos colaterais referentes à síndrome de Cushing e distúrbios metabólicos.

Pode-se dizer, conforme Pauli et al. ${ }^{27}$, que o exercício físico regular, bem orientado para indivíduos que fazem ou vão fazer uso de glicocorticoides, é essencial, levando em consideração as adaptações favoráveis do organismo. Portanto, deve ser associado a outras condutas quando necessárias, beneficiando pacientes asmáticos, portadores de psoríase, atrite reumatoide, lúpus, pênfigo, câncer e outras doenças. 
A relação dos efeitos do exercício físico com a massa óssea e sistemas relacionados, levam a estudos sistêmicos envolvendo diversas formas de atividades físicas, supostamente porque sua prática repercute de diferentes formas, ou seja, resulta em variados estímulos, de acordo com o tipo de exercício.

Apesar de Carvalho ${ }^{12}$ e Haach ${ }^{13}$ apontarem importantes resultados dos efeitos do ultrassom pulsado terapêutico sobre a massa óssea, não se encontrou nenhum trabalho envolvendo exercícios terapêuticos (Fisioterapia) que abordasse o tema ou o norteasse.

\section{Referências}

1. Jacob SW, Francone CA, Lossow WL. Anatomia e fisiologia humana. Rio de Janeiro: Guanabara Koogan; 1990, 5.

2. Wheater PR. Histologia funcional. Rio de Janeiro: Guanabara Koogan; 1994, 3: p. 170-90.

3. Moore KL, Dalley AF. Anatomia orientada para clínica. Rio de Janeiro: Guanabara Koogan. 2001; 4. p. 15-7.

4. Rosen CJ, Bilezikian JP. Anabolic therapy for osteoporosis. J Clin Endocrinol Metab. 2001;86(3):957-64.

5. Schimmer BP, Parker KL. As bases farmacológicas da terapêutica. Mc Grawn Hill; [19--?]: p. 1085-1101.

6. Lanna CMM, Montenegro Junior RM, Pauli FJA. Fisiopatologia da osteoporose induzida por glicocorticóide. Arq Bras Endocrinol Metab. 2003;47(1):9-18.

7. Gregório LH, Locativa, PGS, Melazzi ACC, Russo LAT. Glucocorticoid-induced osteoporosis. Arq Bras Endocrinol Metab. 2006;50(4).

8. Borba VZC, Castro ML. Osteoporose induzida por glicocorticóides. Arq Bras Endocrinol Metab. 1999;43(6):452-45.

9. Lora FL, Amarante HMB, Pisani JC, Borba VVC, Kulak CAM, Carmes ER. Avaliação da densidade mineral óssea em pacientes com doença inflamatória intestinal. Arq Gastroen. 2005;42(4).

10. Azevedo E, Szejnfeld VL. Osteoporose por corticóide. J. Pneumol. 1996,22(6).
11. Pinto AS, Oliveira TT, Del Carlo R, Nagem TJ, Fonseca CC, Moraes GHK et al. Efeitos do tratamento combinado de alendronato de sódio, atorvastatina cálcica e ipriflavona na osteoporose induzida com dexametasona em ratas. Rev Bras Cienc Farm. 2005;26(1):63-70.

12. Carvalho DCL. Ação do ultra-som de baixa intensidade em ossos de ratas osteopênicas [dissertação]. São Carlos: Faculdade de Bioengenharia de São Carlos; 2001.

13. Haach LCA. Utilização do exercício físico versus ultra-som pulsado de baixa intensidade na manutenção de massa óssea [dissertação]. Faculdade de Bioengenharia. São Carlos; 2006.

14. Mcardle WD, Katch FL, Katch, VL. Fisiologia do exercício: energia, nutrição e desempenho humano. Rio de Janeiro: Guanabara Koogan; 2003, 5.

15. Silva CC, Teixeira AS, Goldberg TBL. O esporte e suas implicações na saúde óssea de atletas adolescentes. Rev Bras Med Esp. 2003;9(6).

16. Silva CC, Goldberg TBL, Teixeira AS, Dalmas JC. Análise preditiva da densidade mineral óssea em adolescentes brasileiros eutróficos do sexo masculino. Arq Bras Endocrinol Metab. 2006;50(1).

17. Damiani D, Kuperman H, Dichtchekenian V, Manna TD, Setian N. Corticoterapia e suas repercussões: a relação custo-benefício. Pediatria. 2001;5(1):71-82.

18. Katzung BG. Farmacologia: básica e clínica. Rio de Janeiro: Guanabara Koogan; 2003, 8.

19. Cooper MS. Sensitivity of bone to glucocorticoids. Clinic Science. Birmingham. 2004;107:111-23.

20. Greenspan FS, Strewlwer GJ. Basic and clinical endocrinology. London: Appleton Lange. 1997; 5.

21. Gennari G. Efeito diferencial dos glicocorticóides sobre a absorção de cálcio e sobre a massa óssea. Rev Bras Med. 1994;51(3).

22. Rubin E, Farber JL. Patologia. Rio de Janeiro: Interlivros. 1990: p. 1154-5.

23. Pessan VJO, Volpon JB, Shimano AC. Ensaio mecânico de flexão nas faces côncava e convexa da diáfise do fêmur de ratas. Rev Bras Ortop. 1996;31(7):600-4.

24. Frateschi MEBJM. Efeitos da imobilização e remobilização em algumas propriedades mecânicas do osso [dissertação]. Ribeirão Preto: Universidade de Ribeirão Preto; 2002. 
25. Abrahão GS. Ação da atividade física sobre as propriedades mecânicas de fêmures e tíbias de ratas osteopênicas [dissertação]. Ribeirão Preto: Faculdade de Medicina de Ribeirão Preto; 2004.

26. Carvalho DCL, Rosim GC, Gama LOR, Tavares MR, Tribioli RA, Santos IR. Tratamentos não farmacológicos na estimulação da osteogênese. Rev Saúde Pública. 2002;36(5).

27. Pauli JR, Souza L, Rogatto G, Gomes R, Luciano E. Influência do treinamento físico sobre parâmetros do eixo hipotálamo-pituitária-adrenal de ratos administrados com dexametasona. Rev Port Cien Desp. [19--]:143-52.
28. França SCA, Barros Neto TL, Agresta MC, Lotufo RFM, Kater CE. Resposta divergente da testosterona e do cortisol séricos em atletas masculinos após uma corrida de maratona. Arq Bras Endocrinol Metab. 2006;50(6).

29. Bara Filho MG, Ribeiro LCS, Miranda R, Teixeira MT. A redução dos níveis de cortisol sanguíneo através da técnica de relaxamento progressivo em nadadores. Rev Bras. Med Esp. 2002;8(4):139-43.

30. Canali ES, Kruel LFM. Respostas hormonais ao exercício físico. Rev Paul Educ Fís. 2001;15(2):141-53.

31. Foss ML, Keteyian J. As bases fisiológicas do exercício e do esporte. Guanabara Koogan; 2000, 6. 